\title{
Ultrasensitivity of Cell Adhesion to the Presence of Mechanically Strong Ligands
}

\author{
Mehdi Roein-Peikar, ${ }^{1}$ Qian Xu, ${ }^{2,3}$ Xuefeng Wang, ${ }^{1,2}$ and Taekjip $\mathrm{Ha}^{1,2,3,4, *}$ \\ ${ }^{1}$ Department of Physics and Center for the Physics of Living Cells, \\ University of Illinois at Urbana-Champaign, Urbana, Illinois 61801, USA \\ ${ }^{2}$ Institute for Genomic Biology, University of Illinois at Urbana-Champaign, Urbana, Illinois 61801, USA \\ ${ }^{3}$ Howard Hughes Medical Institute, Urbana, Illinois 61801, USA \\ ${ }^{4}$ Department of Biophysics and Biophysical Chemistry, Department of Biophysics \\ and Department of Biomedical Engineering, Johns Hopkins University, Baltimore, Maryland 21205, USA
}

(Received 8 October 2014; revised manuscript received 4 October 2015; published 5 January 2016)

Integrins, a class of membrane proteins involved in cell adhesion, participate in the cell's sensing of the mechanical environments. We previously showed that, for the initial cell adhesion to occur, single integrins need to experience a threshold force of 40 pico-Newton $(\mathrm{pN})$ through their bond with surface-bound ligands. This force requirement was determined using a series of double-stranded DNA tethers called tension gauge tethers (TGTs), each with a different rupture force, linked to the ligand. Here, we performed cell-adhesion experiments using surfaces coated with two different TGTs, one of a strong rupture force (around $54 \mathrm{pN}$ ) and the other of a weak rupture force (around $12 \mathrm{pN}$ ). When presented with one type of TGT only, cells adhered to the strong TGT-coated surface but not to the weak TGT-coated surface. However, when presented with both, the presence of the strong TGTs transforms the way cells respond to the weak TGTs such that cells treat both TGTs the same, as if the weak TGTs were strong. Furthermore, a subpopulation of cells can adhere to and spread on a surface displaying just a few molecules of the strong TGTs per cell if, and only if, they are presented along with many weak TGTs. This ultrasensitivity to just a few tethers that can withstand strong forces raises a question of how the cells can achieve such remarkable sensitivity to their mechanical environment without amplifying noise.

DOI: 10.1103/PhysRevX.6.011001

\section{INTRODUCTION}

Research into how cells sense and respond to mechanical cues in their environments has shown that the processes of growth, motility, and development are strongly influenced by the mechanical properties of cellular surroundings. Most of these studies have measured the cell's macroscopic, ensemble-averaged response to forces exerted between receptors and their ligands which mediate mechanical communication [1-5]. Relatively few studies have quantified these actions at the molecular level [6-13]. Yet, at the most elementary level, the sensing of the mechanical environment must be performed by single molecules in mechanical contact with the environment, and the cell then must be able to process the single molecular events for its decision making.

One of the best characterized cellular mechanical processes is adhesion to the extracellular matrix (ECM) - the

Corresponding author. tjha@jhu.edu

Published by the American Physical Society under the terms of the Creative Commons Attribution 3.0 License. Further distribution of this work must maintain attribution to the author(s) and the published article's title, journal citation, and DOI.
Subject Areas: Biological Physics, Interdisciplinary Physics microenvironment of animal cells [4,14-16]. The membrane-bound receptor proteins, called integrins, interact with the ECM and relay information about the extracellular environment to the cell interior and to the underlying actin cytoskeleton through interaction with other proteins [11,17-24]. The spatial extent of the ECM communication with the actin cytoskeleton through integrins ranges from nano to micrometers with a force sensitivity that ranges from a few pico-Newton $(\mathrm{pN})$ to a few hundreds of $\mathrm{pN}$ [12,25-31]. Precise understanding of the underlying mechanisms requires techniques that are sensitive in these ranges.

To investigate the single molecular forces involved in mechanical processes in cells, we developed a technique called tension gauge tether (TGT) [32]. This technique leverages the well-understood rupture dynamics of short, double-stranded (ds) DNA, which was previously used to determine the antibody-antigen binding forces [33], in order to determine the magnitude of forces across a single receptor-ligand bond required for triggering certain cellular behaviors, for example, integrin-mediated cell adhesion [32,34], Notch signaling activation [35], and immune cell activation [36]. In a TGT designed for integrins, one strand, termed the top strand, is covalently linked to the RGDfK ligand, which is a short peptide mimic of the ECM [37], 
and the other strand (bottom strand) is covalently linked to a biotin (Fig. 1). The dsDNA is then tethered to a polymerpassivated glass surface through biotin. By keeping the location of the ligand fixed while shifting the location of the biotin progressively away from the ligand, the force required to rupture the dsDNA increases in a quantifiable fashion.

During the cell adhesion process, any bond formed between an integrin and a ligand is thought to be subject to a mechanical force or tension. Several different mechanisms may contribute to this tension, including an active force arising from intracellular processes and a generic physical force associated with the cell membrane. If this force across a single integrin-ligand bond required to initiate stable cell adhesion is greater than the tension tolerance $\left(T_{\text {tol }}\right)$ of the tether, the top strand engaged with an integrin is removed from the surface by the cell-induced rupture of dsDNA. This can be detected as a loss of fluorescence from the surface if the top strand is labeled with a fluorophore. On the other hand, if the required force is smaller than $T_{\text {tol }}$, the integrin will continue to engage with the RGDfK and mediate cell adhesion and spreading. Regardless of how a single integrin is coupled to the membrane, to the underlying cytoskeleton, or to other integrins, the force applied to our DNA tether is applied through a single integrin-ligand bond.

The physics behind short $(<20$ base pairs) dsDNA rupture was originally described by de Gennes [38] and has since been experimentally verified [39]. The equation for the rupture force is the following:

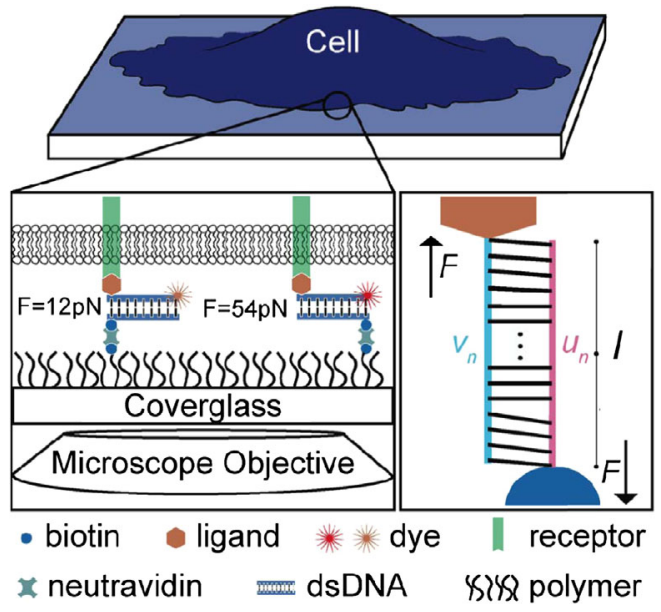

FIG. 1. Schematic of multiplex TGT experiment. Cells cultured are presented with two types of TGTs simultaneously. TGTs are conjugated with the tripeptide RGDfK, which binds to integrin receptors expressed on the cell surface. Each type of TGT is also labeled with a distinct fluorophore, on the top strand, and anchored to the surface through a biotin-neutravidin linkage. The inset shows the DNA tether under tension of magnitude $F$ applied across $l$ base pairs in a shear force configuration.

$$
T_{\mathrm{tol}}=2 f_{c}\left[x^{-1} \tanh \left(\frac{x l}{2}\right)+1\right],
$$

where $T_{\text {tol }}$ is the rupture force, $f_{c}$ is the breaking force of a single base pair, $x$ is related to the spring constant of DNA, and $l$ is the number of base pairs (bp) between the points of force application on the two complementary strands of DNA. In our case, $l$ represents the number of bp separating RGDfK and biotin (Fig. 1 inset). Using magnetic tweezers, the Prentiss group determined the values for $x^{-1}$ and $f_{c}$ to be $6.8 \mathrm{bp}$ and $3.9 \mathrm{pN}$, respectively [39]. On a DNA with a total length of $18 \mathrm{bp}$, when the RGDfK and biotin are placed closest to each other ( $l=1 \mathrm{bp}), T_{\text {tol }}$ is $\sim 12 \mathrm{pN}$, whereas $T_{\text {tol }}$ is $\sim 54 \mathrm{pN}$ when the biotin is placed farthest away from RGDfK ( $l=18 \mathrm{bp}$ ). Because the magnetic tweezer experiments that yielded the parameters for the de Gennes model applied a constant force for 1 or 2 seconds and increased the force incrementally until the DNA ruptured, the $T_{\text {tol }}$ values we estimate should be considered only approximate. The absolute force values may be different from our estimates if the cellular time scale of force application is much longer or shorter than 1 to 2 seconds.

Prior TGT studies revealed the tension threshold for integrin-mediated cell adhesion to be about $40 \mathrm{pN}$ [32]. In these experiments, TGT with nine different $T_{\text {tol }}$ values ranging from $12 \mathrm{pN}$ to $54 \mathrm{pN}$ were individually presented to cells. After a 30-minute incubation, cells did not adhere stably to the surface if TGT with $T_{\text {tol }}<43 \mathrm{pN}$ was used. On the other hand, cells adhered stably if TGT with $T_{\text {tol }} \geq$ $43 \mathrm{pN}$ was used. The tension threshold appeared universal across several different cell types, both cancerous and noncancerous [32,34], and was shown to hold as early as the first 5 minutes of adding the cells to the TGT-coated surface [32]. This $40 \mathrm{pN}$ force across a single bond outside the cell may contribute to the range of single molecule forces, $5 \mathrm{pN}-25 \mathrm{pN}$, proposed to be experienced by the intracellular proteins that bridge integrins to the actin cytoskeleton [11,17,40,41].

The ECM is complex, presenting a great variety of ligands with which integrins can interact. Our objective in this work is to better approximate this complexity and extend the prior work, which quantified cellular response to only one tether strength at a time. By presenting both weak $\left(T_{\text {tol }} \sim 12 \mathrm{pN}\right)$ and strong $\left(T_{\text {tol }} \sim 54 \mathrm{pN}\right)$ tethers to cells simultaneously in a scheme we call "TGT multiplexing (MP)" (Fig. 1), we can study cell mechanics at the molecular level in an environment that is more similar to the ECM.

\section{RESULTS AND DISCUSSION}

\section{A. Multiplexing weak and strong TGTs}

In the multiplex scheme, weak and strong TGTs are made distinguishable from each other through labeling with 
fluorophores of different colors, $\mathrm{Cy} 3$ and $\mathrm{Cy} 5$, respectively. A typical experiment arrays three circular spots of TGT on the same imaging surface, each with an area of about $13 \mathrm{~mm}^{2}$ and a surface density of about 400 tethers $/ \mu \mathrm{m}^{2}$, which mirrors the density of integrins in the membrane of animal cells $\left(40-3000 / \mu \mathrm{m}^{2}\right)$ [42]. To achieve these conditions, $3 \mu \mathrm{L}$ of a $1 \mu \mathrm{M}$ TGT solution is applied onto a neutravidin functionalized glass surface. After a 10-minute incubation period, unbound TGTs are thoroughly rinsed away.

The first spot presents only the weak TGT, and the second presents only the strong TGT. The third spot presents multiplexed weak and strong TGT which are mixed at equimolar concentrations. Chinese Hamster Ovary (CHO-K1, American Type Cell Culture) cells are cultured on the surface for 30 minutes in a $37^{\circ} \mathrm{C}$ incubator. Afterwards, unbound cells are gently rinsed away, and the sample is fixed. Imaging the cells using differential interference contrast (DIC) and epifluorescence microscopy (Zeiss 200M Axiovert) allowed us to obtain two main observables from the images: (1) the degree of TGT rupture reported through loss of fluorescence from the imaging surface and (2) the number of cells that remain attached. Very few cells remained attached after gentle washing when only the weak TGT was used, whereas a high density of adherent cells was observed with the strong TGT alone or with TGT multiplexing. To ensure that fixation does not bias the results, experiments have been performed using both live and fixed cells. In the context of these two observables, there were no noticeable differences between fixed and live cell images (not shown).

\section{B. Multiplexing transforms how the cells treat the weak tethers}

Fluorescence images from a typical experiment are shown in Fig. 2. Similar to what was previously reported [32], fluorescence images of the weak TGT-coated surface showed uniformly dark patches about the size of single cells, likely due to the rupture of weak TGTs induced by cellular forces transmitted through integrins when the cell tries to gain a foothold by pulling on ligands [Fig. 2(a)]. Imaging the cells without rinsing confirmed this interpretation because fluorescence loss was observed only under the cells (not shown). Cellular forces that ruptured the weak TGTs are likely provided by actin cytoskeleton instead of passive sources such as membrane repulsion because an actin filament inhibitor $(1-\mu \mathrm{M}$ latrunculin $\mathrm{A})$ eliminated fluorescence loss [Fig. 2(a)]. On the other hand, the strong TGT-coated surface showed lower degrees of rupture, with scattered dark regions localized to the periphery of the contact area between the cell and the surface [Fig. 2(b)]. The peripheral (or edge) rupture of the strong TGT appears to be caused by an ATP-powered molecular motor, myosin II (Wang et al. [43]). When weak and strong TGTs are multiplexed and presented to cells simultaneously, the cells

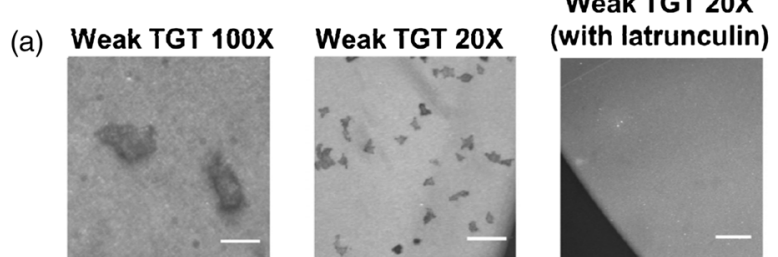

(b) Strong TGT 100X

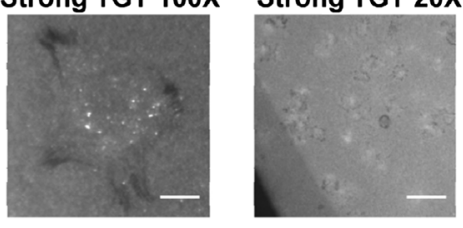

(c)

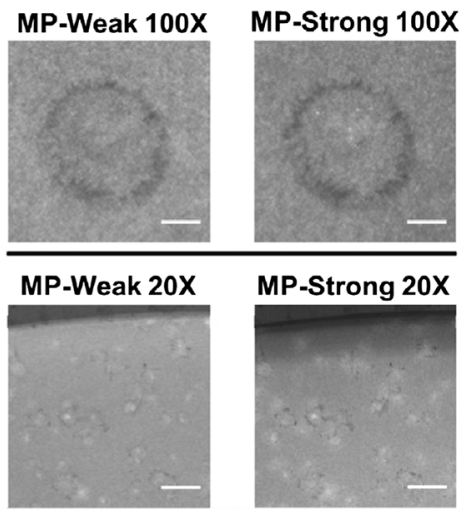

FIG. 2. Fluorescence images of TGT rupture with and without multiplexing. (a) Fluorescent images on the weak TGT-coated surface in 20X and 100X magnifications show uniformly dark patches beneath the cell (uniform rupture). However, when we inhibited actin polymerization by adding $1 \mu \mathrm{M}$ latrunculin $\mathrm{A}$ to the cell culture medium, cells did not show rupture footprint. (b) Fluorescent images on the strong TGT-coated surface show fluorescence loss mostly at the periphery of the cell (edge rupture). Bright spots are probably the vesicles containing the upper strand of ruptured TGT that have undergone endocytosis. (c) Fluorescence loss patterns of weak and strong TGTs are similar and show "edge rupture" on the multiplex TGT surface. Scale bars are $50 \mu \mathrm{m}$ for $20 \mathrm{X}$ and $10 \mu \mathrm{m}$ for $100 \mathrm{X}$.

adhered well to the surface after rinsing, as was the case for the strong TGT-coated surface. Unexpectedly, the rupture patterns for the weak TGT changed, becoming identical to those observed for the strong TGT. In other words, instead of the uniformly dark rupture footprint for the weak TGTcoated surface, cells ruptured both tethers on the periphery of the contact area, as if they were both strong TGTs [Fig. 2(c)]. These observations suggest that the presence of strong tethers can significantly influence how cells sense the weak tethers and act on them.

\section{Multivariable single-cell analysis}

We further quantified rupture footprint patterns by analyzing more than 100 cells from each surface in terms of two variables [Fig. 3(a)]. The first variable is the degree 
of TGT rupture. This is measured by comparing the fluorescence intensity underneath a cell to a nearby surface without any attached cells. The background, which is measured from an area off of the TGT spot, is subtracted from both values before the comparison. To calculate this, the following formula is used:

$$
\text { Rupture }(\%)=\frac{\mathrm{MF}_{\text {surface }}-\mathrm{MF}_{\text {cell }}}{\mathrm{MF}_{\text {surface }}-\mathrm{MF}_{\text {background }}}
$$

where MF is the mean fluorescence intensity. Values are measured in ImageJ (open-source software developed by the National Institutes of Health) by selecting the corresponding region. For the weak TGT spot, regions were selected directly from the fluorescence images since this surface does not retain cells after rinsing. For the rest of the spots, DIC microscopy was used to image the cells directly. The regions for analysis were selected from these DIC images, and the corresponding fluorescence images were analyzed.

Because the analysis of the images from the weak TGT spot is different from the analysis for the other two spots, we performed a control comparison to rule out the possibility of any analytical bias. For the strong TGT, we selected regions in two different ways. First, regions were selected directly from the fluorescence images and the percent rupture was calculated. Second, regions were selected from DIC images and the percent rupture was calculated based on the corresponding fluorescence images. The values for the percent rupture from the two analyses were within a standard error of each other: $8.50 \% \pm 1.01$ and $8.27 \% \pm 0.99$, respectively.

The second variable describes the spatial distribution of the rupture. In other words, underneath the cell, does the rupture occur everywhere or is it concentrated in a specific area? As a quantitative measure, we define a rupture moment, $I$, analogous to the moment of inertia from mechanics. A bigger value of $I$ represents rupture at the periphery of the contact area underneath each cell, or "edge rupture." A smaller value suggests that the rupture is more uniformly localized, or "uniform rupture."

We used the following formula:

$$
I=\frac{\sum_{i=1}^{N} M_{i} R_{i}^{2}}{A \sum_{i=1}^{N} M_{i}}
$$

where $A$ is the area of the cell measured in Image J, $R_{i}$ is the distance from the approximate center of the fluorescence footprint underneath a single cell to the $i$ th pixel, $M_{i}$ is the percent rupture of the $i$ th pixel as defined in Eq. (2), and $N$ is the total number of pixels.

A scatter plot of the percent rupture versus $I$ for many single cells [Fig. 3(a)] shows that weak TGT and strong TGT rupture patterns form two distinct clusters. Cells showed higher rupture percentage and smaller $I$ on the (a)

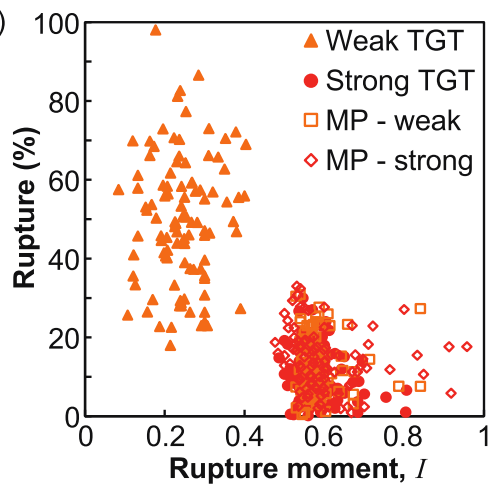

(b)

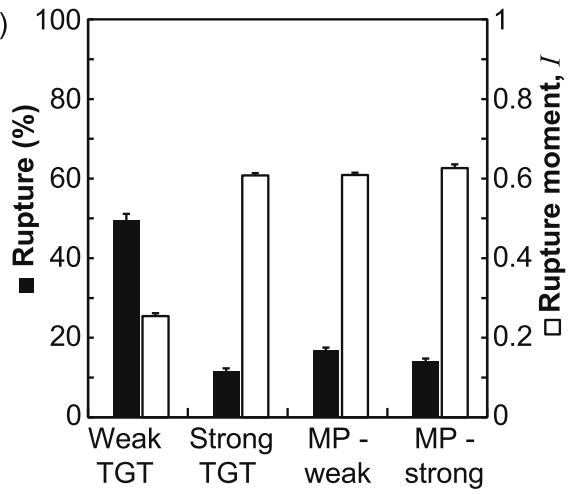

FIG. 3 Single cell analysis of TGT rupture pattern. (a) More than 400 cells are selected and analyzed based on two variables: percent rupture and rupture moment $(I)$. Each symbol represents one single cell. Percent rupture and rupture moment for the weak TGT-coated surface (Weak TGT) are characteristic of "uniform rupture" and are clearly different from "edge rupture" characteristics seen for the strong TGT surface (Strong TGT), and for the weak and strong TGTs on the MP surface (MP-weak and MPstrong, respectively). (b) Average values from (a). Error bars denote standard errors of mean.

weak TGT-coated surface compared to the strong TGTcoated surface. On the multiplex (MP) surface, the rupture patterns of both weak and strong TGTs cluster together with each other and with that of the strong TGT-coated surface, showing, indeed, that the presence of strong TGTs transforms the way cells treat the weak TGTs. The ensemble average values of rupture percentage and $I$ [Fig. 3(b)] further support the qualitative observations shown in Fig. 2. Taken altogether, cells act on both tethers similarly in MP, showing small-percent rupture values and large $I$ values, which are characteristics of edge rupture, as if both tethers are strong.

\section{Multiplexing leads to ultrasensitivity for strong TGT}

Up to this point, TGT spots have been prepared with high surface densities so that each cell would have $>200,000$ TGT molecules underneath, on average. This number is estimated from the known surface density given $1 \mu \mathrm{M}$ incubation concentrations (about $400 \mathrm{TGT} / \mu \mathrm{m}^{2}$ ) [34] and the measured average area of cells attached to 
the strong TGT and MP spots (about $600 \mu \mathrm{m}^{2}$ ). For a point of reference, the aerial footprint of CHO-K1 cells used in our experiments ranges from $80 \mu \mathrm{m}^{2}$ (for the cells that do not adhere to the surface with weak tethers and just leave a fluorescent footprint) to $800 \mu \mathrm{m}^{2}$ (for the cells adhered to the surface with strong tethers).

Here, we define the term, "weak tether transformation" to refer to the shift from the uniform rupture of weak tethers, when presented alone, to edge rupture when the weak tethers are multiplexed with the strong ones. A question then arises: How many strong tethers are needed for the cell to undergo a weak tether transformation? To answer this question, we progressively lowered the incubation concentration of strong TGT from $1 \mu \mathrm{M}$ to $0 \mathrm{pM}$ while maintaining the weak TGT concentration during incubation at $1 \mu \mathrm{M}$. Cells were cultured on these MP titration spots for 30 minutes, and then rinsed, fixed, and imaged. The images were analyzed, as before, in terms of (1) the number of cells stably adhered per unit area and (2) rupture pattern (the rupture percentage and I). As a control, strong TGT is presented alone to cells over the same range of concentrations.

Figure 4(a) shows representative DIC and fluorescence images of the MP surface obtained with $1 \mu \mathrm{M}$ weak TGT and $40 \mathrm{nM}, 2 \mathrm{pM}$ and $0 \mathrm{pM}$ strong TGT. Very few cells adhered to the surface in the absence of any strong TGT, but even at 2 pM strong TGT, 500,000 fold dilution compared to weak TGT, we observed many cells adhering, albeit with lower cell counts than in the case of $40 \mathrm{nM}$ strong TGT [Figs. 4(a) and 4(b)]. In addition, the fluorescence rupture pattern for the weak TGT underneath adhered cells for both $2 \mathrm{pM}$ and $40 \mathrm{nM}$ TGT showed edge rupture, indicating weak tether transformation.

Figure 4(b) shows the plot of adhered cell count. When cells are cultured on the strong TGT-coated surface, only background levels of adhered cells are observed for concentrations lower than $40 \mathrm{nM}$. However, when weak tethers are present, cells adhere for strong TGT concentration down to $2 \mathrm{pM}$ but not at $0.2 \mathrm{pN}$. The attached cell count, although well above background levels, decreased on MP titration spots with pM concentrations of the strong TGT. Overall, our data suggest that even at $2 \mathrm{pM}$ incubation concentration, strong TGT can induce weak tether transformation for a subset of cells.

We further analyzed the weak TGT rupture patterns under adhered cells and calculated the rupture percentage and $I$ from single cells [Fig. 5]. At $2 \mathrm{pM}$ and $2 \mathrm{nM}$ incubating concentrations of strong TGTs, although some of the loss-of-fluorescence footprints show uniform rupture, these footprints do not correspond to adhered cells in the DIC images. Instead, the footprints for adhered cells show edge rupture patterns as quantified through rupture percentage and $I$ [Fig. 5(b)] at strong TGT concentrations of $2 \mathrm{pM}$ or above.
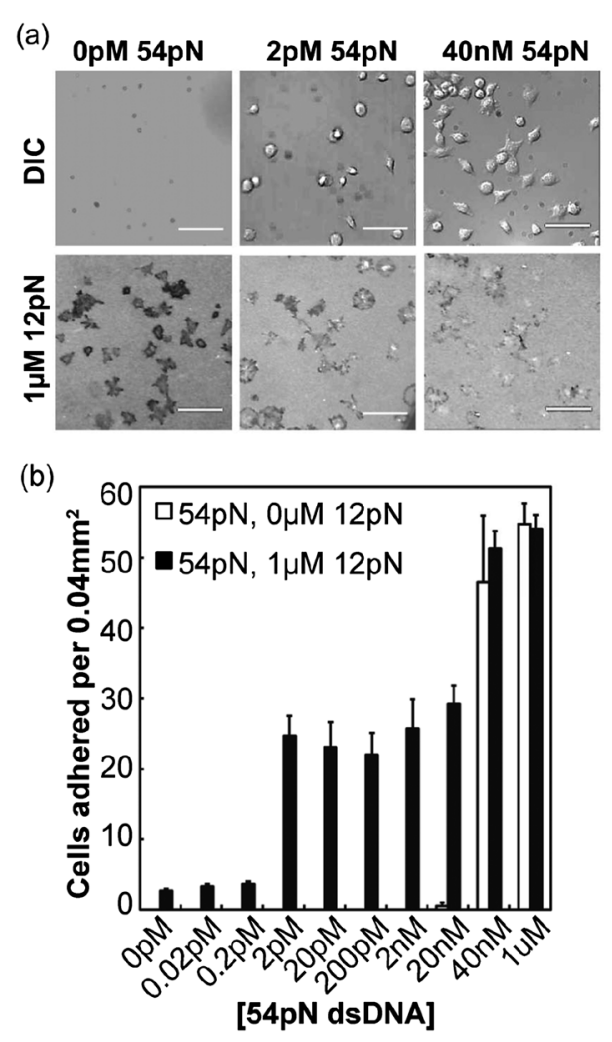

FIG. 4. Titration of strong TGT in multiplexing experiment. (a) Representative images of cells on different MP TGT surfaces. Differential interference contrast (DIC, top) and fluorescence loss of the weak TGT (bottom). Scale bars are $50 \mu \mathrm{m}$. (b) Average density of adherent cells versus strong TGT incubation concentration. Only background levels of adhered cells are observed for concentrations lower than $40 \mathrm{nM}$ of strong TGTs alone. However, when weak tethers are present, cells adhered for strong TGT concentration down to $2 \mathrm{pM}$ but not at $0.2 \mathrm{pN}$. The cell density, although well above background levels, decreased for $\mathrm{pM}$ concentrations of the strong TGT. Error bars denote standard errors of mean.

At incubation concentrations $\leq 200 \mathrm{pM}$, the average surface density of strong TGT in the presence of unlabeled $1 \mu \mathrm{M}$ of weak TGT can be directly determined using single-molecule total-internal-reflection fluorescence microscopy. From these measurements, we estimated the number of strong TGT per cell as a function of the pM incubation concentrations using $600 \mu \mathrm{m}^{2}$ as the average area of an adhered cell [Fig. 6(a)]. Note that the surface density of strong tethers is 3 times lower when they are presented together with weak tethers, probably because weak and strong tethers can compete with each other for a finite number of binding sites on the surface.

According to our calibration, the number of strong tethers at $2 \mathrm{pM}$ incubation is about two molecules per cell, suggesting that, even with the uncertainty in the measurement, the number of strong tethers required for weak tether transformation is in the range of low single digits per cell, possibly down to one strong tether. This 


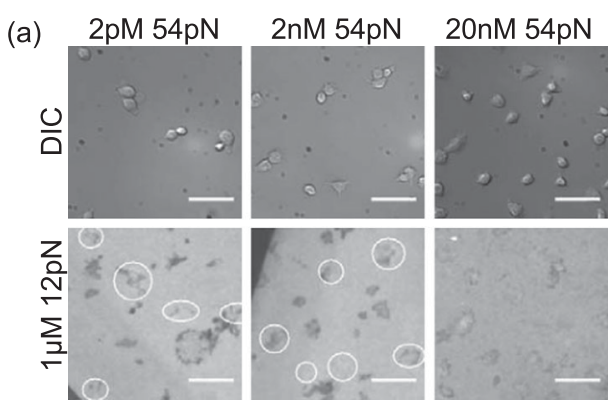

(b)

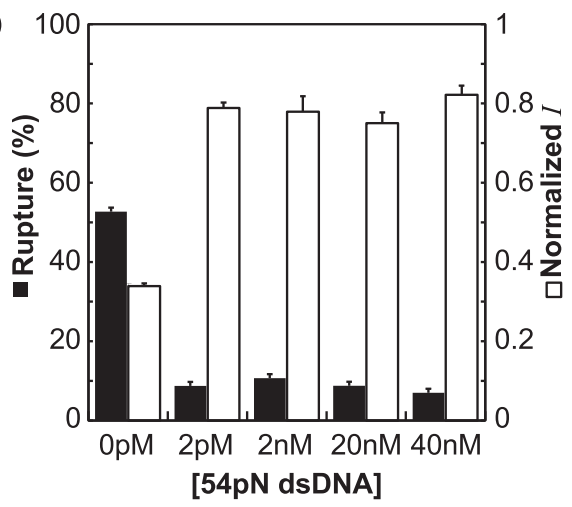

FIG. 5. Weak tether transformation vs strong TGT concentration. (a) Representative images of MP TGT surfaces. DIC (top) and fluorescence loss of the weak TGT (bottom). The white outlined regions in the bottom images correspond to adhered cells in the top images. Scale bars are $50 \mu \mathrm{m}$. (b) Percent rupture and normalized rupture moment $(I)$ are measured and averaged for cells vs strong TGT concentration. Error bars denote standard errors of mean.

ultrasensitivity for strong tethers is a surprising result. Cells do not adhere to a surface displaying either weak tethers alone or strong tethers at the $\mathrm{pM}$ incubation concentrations. Yet, the presence of just a few strong tethers per cell will induce adhesion if, and only if, the surface is also displaying many weak tethers. We can rule out the possibility that the strong tether distribution is highly uneven, leading to localized "hot spots" that display many tethers, because direct single-molecule microscopy measurements revealed no such unevenness [Fig. 6(b)].

Because the adhered cell count on the MP surfaces with picomolar strong tether concentrations is lower than on those with $\geq 20 \mathrm{nM}$ concentrations of strong tethers [Fig. 4(b)], we hypothesize that there exists a subpopulation of ultrasensitive cells that can be fully activated by just a few strong tethers, leading to adhesion and spreading. As the amount of strong tethers is increased from $2 \mathrm{pM}$ to 2 $\mathrm{nM}$, the adhered cell count is unchanged because only ultrasensitive cells adhere. At $\geq 20 \mathrm{nM}$, cell attachment count increases because the less sensitive cell population is then activated.

In summary, we report synergistic mechanical forces in cellular adhesion using TGT multiplexing. Multiplexing a strong tether with a weak tether resulted in two surprising (a)

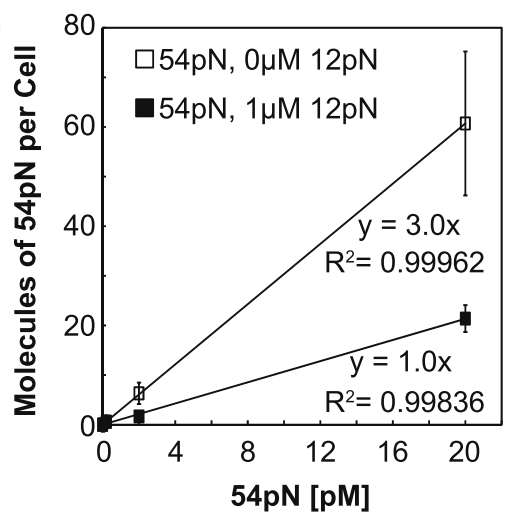

(b)

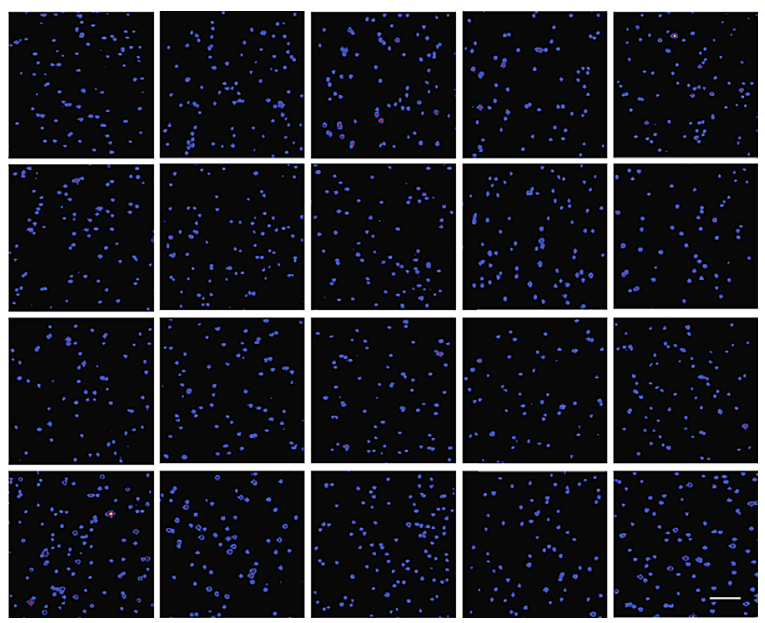

FIG. 6. Single molecule measurements of strong TGT density. (a) At picomolar incubation concentrations, the average surface densities of strong TGT in the presence of unlabeled $1 \mu \mathrm{M}$ of weak TGT were directly determined using single-molecule totalinternal-reflection fluorescence microscopy. From these measurements, the number of strong TGT per cell as a function of the $\mathrm{pM}$ incubation concentrations was estimated. Note that the surface density of strong tethers is 3 times lower when they are presented together with weak tethers, probably because weak and strong tethers can compete with each other for a finite number of binding sites on the surface. (b) 20 single-molecule images of DNA tethers $(50 \mathrm{pM}$ Cy5-labeled strong TGTs and $1 \mu \mathrm{M}$ unlabeled weak TGTs during incubation) show that there is no nonrandom clustering of tethers. The scale bar in the lower right image is $5 \mu \mathrm{m}$.

observations. First, the way cells sense and treat the weak tether is transformed in the multiplex scenario: Cells treat both TGTs the same, as if the weak tether were strong. And second, cells adhere to a MP spot when merely a few single molecules of the strong tether are present per cell, as long as there are also many weak tethers.

Although there is a precedent for ultrasensitivity in immune cells where even a single cognate ligand is able to activate the immune cell in the presence of a large amount of noncognate ligands [44], our work is distinct in that cells can change their behavior based on purely mechanical differences. Both strong and weak TGTs 
present the chemically identical ligands to the cells, with the only difference being the mechanical stability of the tethers.

What might be the underlying mechanisms for the remarkable sensitivity to the presence of a minute number of mechanically strong ligands? We previously presented evidence that the tension threshold for cell adhesion can be reduced by lowering the membrane tension [32]. Therefore, processes that modulate the dynamics of the cell membrane and the actin cytoskeleton linked to the membrane may be important in the observed ultrasensitivity to strong forces. There is evidence that cell adhesion is aided by transient contacts and force generation induced by membrane undulations [45]. In addition, sugar-protein coating called glycocalyx on cell membranes may exert steric repulsion force between the cell and the ECM and may thus influence cell adhesion [46-48]. Therefore, we offer one possible explanation for our observation, as follows. Once a cell establishes a link to a ligand on strong TGT, this stable link would lower the membrane in the surrounding area, dampening membrane fluctuations and expelling glycocalyx, which normally separates the membrane from the ECM. Glycocalyx expulsion would then lead to the recruitment of more integrins to form integrin clusters [46], making nearby ligands on weak TGTs more accessible to integrins. The dampening of membrane fluctuations and associated forces would prevent the rupture of weak tethers, and the area of close contact initiated by a single strong TGT may then expand. In other words, a very small number of strong TGTs may form individual nuclei, ultimately leading to cell adhesion. In addition, the integrins recruited around the strong TGTs may become activated to obtain much higher affinity to the ligands [49]. If there are only weak tethers, membrane fluctuations would rupture weak TGTs so that stable adhesion cannot be nucleated. If there are only a few strong tethers, the initial stable contact cannot expand and cells cannot adhere.

We have also observed ultrasensitivity from melanoma cells (B16-F1) in addition to CHO cells, but several other cell types we tested did not show this property. Future studies employing other cell types and biological perturbation tools may be able to reveal the underlying mechanisms that set certain cells apart in terms of their ultrasensitivity to single molecular forces. Regardless of the detailed mechanisms, our present data are consistent with the following time courses of single integrin-ligand bonds that form during initial cell adhesion. When a single integrin-ligand bond forms, the cell gradually increases the force across the bond to about $40 \mathrm{pN}$ so that weak TGTs rupture. When the force reaches about $40 \mathrm{pN}$ for just a few integrins bound through strong TGTs, the cell apparently makes a decision that the surface is rigid enough for adhesion and the force across the strong TGTs drops to a low value. Subsequent bonds only experience this low steady-state force so that the weak TGTs do not rupture
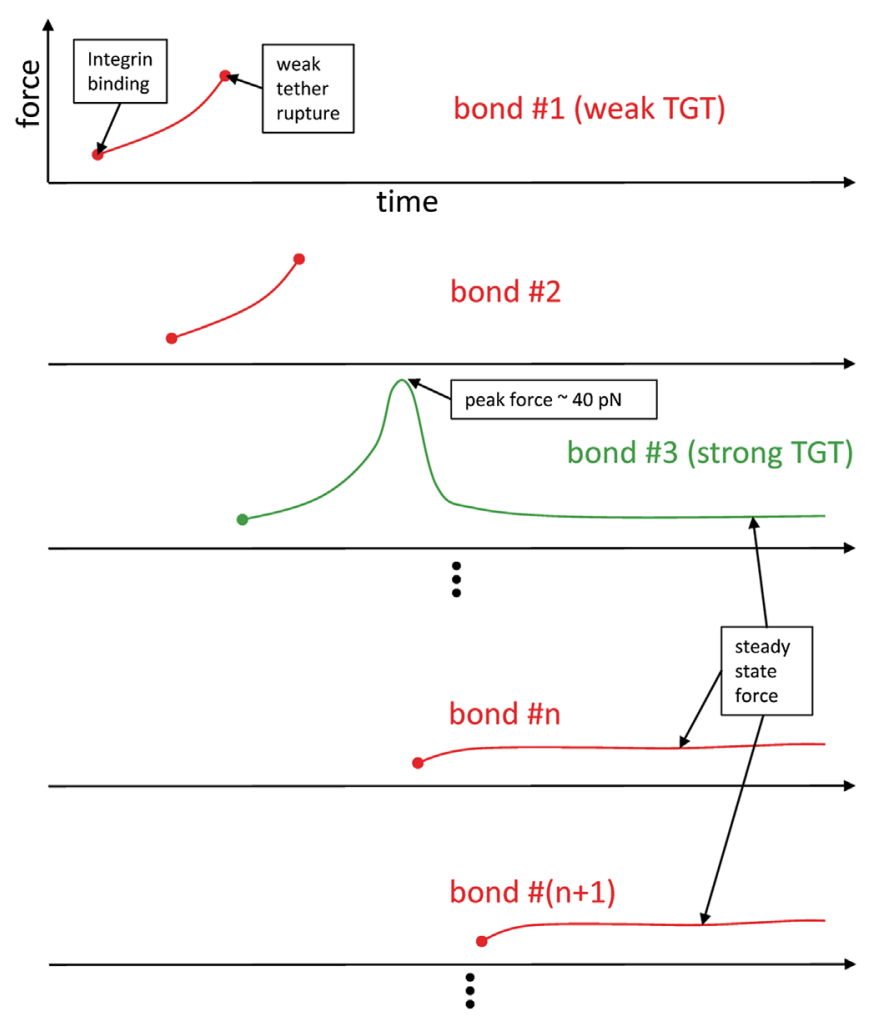

FIG. 7. Proposed time courses of mechanical engagements through single integrin-ligand bonds. A cell grabs a ligand attached to the surface through a weak tether and applies gradually increasing force until the weak tether ruptures. If a strong tether is pulled, the $40 \mathrm{pN}$ threshold force for adhesion is reached, telling the cell that the substrate is rigid enough for adhesion. Then, the force through the bond drops to a low, steadystate value, and subsequent bonds experience only this lower force. The cell no longer needs to apply strong forces because it has already determined that the substrate is rigid enough.

anymore (Fig. 7). In this model, rigidity sensing of the underlying surface $[2,50-60]$ can be completed by just a few integrin-ligand bonds, raising an interesting question of how the cell achieves such mechanical ultrasensitivity without amplifying noise. Future studies utilizing live cell imaging with high space and time resolution and high sensitivity may be able to test various aspects of this model.

\section{ACKNOWLEDGEMENTS}

We thank Erich Sackmann, Brian T. Mulcahy, Ning Wang, Farhan Chowdhury, and Kyu Young Han for a careful reading of the manuscript and for helpful discussions and suggestions. Funding for this research was provided by the National Science Foundation through the Physics Frontiers Center program (PHY 1430124). T. H. is an investigator at the Howard Hughes Medical Institute.

M. R.-P. and Q.X. contributed equally to this work. 
[1] D. E. Ingber, Cellular Mechanotransduction: Putting All the Pieces Together Again, FASEB J. 20, 811 (2006).

[2] A. J. Engler, S. Sen, H. L. Sweeney, and D. E. Discher, Matrix Elasticity Directs Stem Cell Lineage Specification, Cell 126, 677 (2006).

[3] B. D. Hoffman, C. Grashoff, and M. A. Schwartz, Dynamic Molecular Processes Mediate Cellular Mechanotransduction, Nature (London) 475, 316 (2011).

[4] F. M. Watt and W. T. S. Huck, Role of the Extracellular Matrix in Regulating Stem Cell Fate, Nature Reviews, Mol. Cell Biol. 14, 467 (2013).

[5] P. W. Oakes and M. L. Gardel, Stressing the Limits of Focal Adhesion Mechanosensitivity, Curr. Opin. Cell Biol. 30, 68 (2014).

[6] M. Morimatsu, A. H. Mekhdjian, A. S. Adhikari, and A. R. Dunn, Molecular Tension Sensors Report Forces Generated by Single Integrin Molecules in Living Cells, Nano Lett. 13, 3985 (2013).

[7] Y. Liu, K. Yehl, Y. Narui, and K. Salaita, Tension Sensing Nanoparticles for Mechano-Imaging at the Living/ Nonliving Interface, J. Am. Chem. Soc. 135, 5320 (2013).

[8] M. Morimatsu, A. H. Mekhdjian, A. C. Chang, S. J. Tan, and A.R. Dunn, Visualizing the Interior Architecture of Focal Adhesions with High-Resolution Traction Maps, Nano Lett. 15, 2220 (2015).

[9] C. Jurchenko, Y. Chang, Y. Narui, Y. Zhang, and K. S. Salaita, Integrin-Generated Forces Lead to StreptavidinBiotin Unbinding in Cellular Adhesions, Biophys. J. 106, 1436 (2014).

[10] Y. Zhang, C. H. Ge, C. Zhu, and K. Salaita, DNA-Based Digital Tension Probes Reveal Integrin Forces During Early Cell Adhesion, Nat. Commun. 5, 5167 (2014).

[11] C. Grashoff et al., Measuring Mechanical Tension across Vinculin Reveals Regulation of Focal Adhesion Dynamics, Nature (London) 466, 263 (2010).

[12] D. R. Stabley, C. Jurchenko, S. S. Marshall, and K. S. Salaita, Visualizing Mechanical Tension across Membrane Receptors with a Fluorescent Sensor, Nat. Methods 9, 64 (2012).

[13] W. R. Gordon et al., Mechanical Allostery: Evidence for a Force Requirement in the Proteolytic Activation of Notch, Dev. Cell 33, 729 (2015).

[14] G. Halder, S. Dupont, and S. Piccolo, Transduction of Mechanical and Cytoskeletal Cues by YAP and TAZ, Nat. Rev. Mol. Cell Biol. 13, 591 (2012).

[15] H. G. Dobereiner, B. Dubin-Thaler, G. Giannone, H. S. Xenias, and M. P. Sheetz, Dynamic Phase Transitions in Cell Spreading, Phys. Rev. Lett. 93, 108105 (2004).

[16] E. A. Evans and D. A. Calderwood, Forces and Bond Dynamics in Cell Adhesion, Science 316, 1148 (2007).

[17] A. del Rio, R. Perez-Jimenez, R. Liu, P. Roca-Cusachs, J. M. Fernandez, and M. P. Sheetz, Stretching Single Talin Rod Molecules Activates Vinculin Binding, Science 323, 638 (2009).

[18] Y. Sawada, M. Tamada, B. J. Dubin-Thaler, O. Cherniavskaya, R. Sakai, S. Tanaka, and M. P. Sheetz, Force Sensing by Mechanical Extension of the SRC Family Kinase Substrate p130Cas, Cell 127, 1015 (2006).
[19] J. C. Friedland, M. H. Lee, and D. Boettiger, Mechanically Activated Integrin Switch Controls $\alpha_{5} \beta_{1}$ Function, Science 323, 642 (2009).

[20] Y. Wang, E. L. Botvinick, Y. Zhao, M. W. Berns, S. Usami, R. Y. Tsien, and S. Chien, Visualizing the Mechanical Activation of SRC, Nature (London) 434, 1040 (2005).

[21] J. L. Guan, Role of Focal Adhesion Kinase in Integrin Signaling, Int. J. Biochem. Cell Biol. 29, 1085 (1997).

[22] L. Y. Koo, D. J. Irvine, A. M. Mayes, D. A. Lauffenburger, and L.G. Griffith, Co-regulation of Cell Adhesion by Nanoscale RGD Organization and Mechanical Stimulus, J. Cell Sci. 115, 1423 (2002).

[23] S. I. Fraley, Y. F. Feng, R. Krishnamurthy, D. H. Kim, A. Celedon, G. D. Longmore, and D. Wirtz, A Distinctive Role for Focal Adhesion Proteins in Three-Dimensional Cell Motility, Nat. Cell Biol. 12, 598 (2010).

[24] J. T. Parsons, A. R. Horwitz, and M. A. Schwartz, Cell Adhesion: Integrating Cytoskeletal Dynamics and Cellular Tension, Nat. Rev. Mol. Cell Biol. 11, 633 (2010).

[25] N. Wang, J. P. Butler, and D. E. Ingber, Mechanotransduction across the Cell Surface and through the Cytoskeleton, Science 260, 1124 (1993).

[26] P. P. Lehenkari and M. A. Horton, Single Integrin Molecule Adhesion Forces in Intact Cells Measured by Atomic Force Microscopy, Biochem. Biophys. Res. Commun. 259, 645 (1999).

[27] E. A. Cavalcanti-Adam, T. Volberg, A. Micoulet, H. Kessler, B. Geiger, and J.P. Spatz, Cell Spreading and Focal Adhesion Dynamics Are Regulated by Spacing of Integrin Ligands, Biophys. J. 92, 2964 (2007).

[28] G. Jiang, G. Giannone, D. R. Critchley, E. Fukumoto, and M. P. Sheetz, Two-Piconewton Slip Bond between Fibronectin and the Cytoskeleton Depends on Talin, Nature (London) 424, 334 (2003).

[29] J. L. Tan, J. Tien, D. M. Pirone, D. S. Gray, K. Bhadriraju, and C. S. Chen, Cells Lying on a Bed of Microneedles: An Approach to Isolate Mechanical Force, Proc. Natl. Acad. Sci. U.S.A. 100, 1484 (2003).

[30] B. L. Blakely et al., A DNA-Based Molecular Probe for Optically Reporting Cellular Traction Forces, Nat. Methods 11, 1229 (2014).

[31] J. Kim, N. E. Hudson, and T. A. Springer, Force-Induced On-Rate Switching and Modulation by Mutations in Gain-of-Function von Willebrand Diseases, Proc. Natl. Acad. Sci. U.S.A. 112, 4648 (2015).

[32] X. Wang and T. Ha, Defining Single Molecular Forces Required to Activate Integrin and Notch Signaling, Science 340, 991 (2013).

[33] C. Albrecht et al., DNA: A Programmable Force Sensor, Science 301, 367 (2003).

[34] F. Chowdhury et al., Single Molecular Force across Single Integrins Dictates Cell Spreading, Integr. Biol. 7, 1265 (2015).

[35] S. Cocco, R. Monasson, and J. F. Marko, Force and Kinetic Barriers to Unzipping of the DNA Double Helix, Proc. Natl. Acad. Sci. U.S.A. 98, 8608 (2001).

[36] Z. Wan et al., The Activation of IgM- or Isotype-Switched IgG- and IgE-BCR Exhibits Distinct Mechanical Force Sensitivity and Threshold, eLife 4, e06925 (2015). 
[37] Y. Takada, X. Ye, and S. Simon, The Integrins, Genome Biol. 8, 215 (2007).

[38] P. G. de Gennes, Maximum Pull Out Force on DNA Hybrids, C.R. Acad. Sci., Ser. IV 2, 1505 (2001).

[39] K. Hatch, C. Danilowicz, V. Coljee, and M. Prentiss, Demonstration that the Shear Force Required to Separate Short Double-Stranded DNA Does Not Increase Significantly with Sequence Length for Sequences Longer Than 25 Base Pairs, Phys. Rev. E 78, 011920 (2008).

[40] M. Yao, B. T. Goult, H. Chen, P. Cong, M. P. Sheetz, and J. Yan, Mechanical Activation of Vinculin Binding to Talin Locks Talin in an Unfolded Conformation, Sci. Rep. 4, 4610 (2014).

[41] L. Rognoni, J. Stigler, B. Pelz, J. Ylanne, and M. Rief, Dynamic Force Sensing of Filamin Revealed in SingleMolecule Experiments, Proc. Natl. Acad. Sci. U.S.A. 109, 19679 (2012).

[42] P. W. Wiseman, C. M. Brown, D. J. Webb, B. Hebert, N. L. Johnson, J. A. Squier, M. H. Ellisman, and A. F. Horwitz, Spatial Mapping of Integrin Interactions and Dynamics During Cell Migration by Image Correlation Microscopy, J. Cell Sci. 117, 5521 (2004).

[43] X. Wang, J. Sun, Q. Xu, F. Chowdhury, M. Roein-Peikar, Y. Wang, and T. Ha, Integrin molecular tension within motile focal adhesions, Biophys. J. 109, 2259 (2015).

[44] M. J. Yuri Sykulev, I. Vturina, T. J. Tsomides, H. N. Eisen, Evidence that a Single Peptide-MHC Complex on a Target Cell Can Elicit a Cytolytic T Cell Response, Immunity $\mathbf{4}$, 565 (1996).

[45] A. Pierres, V. Monnet-Corti, A. M. Benoliel, and P. Bongrand, Do Membrane Undulations Help Cells Probe the World?, Trends Cell Biol. 19, 428 (2009).

[46] M. J. Paszek et al., The Cancer Glycocalyx Mechanically Primes Integrin-Mediated Growth and Survival, Nature (London) 511, 319 (2014).

[47] R. Bruinsma, A. Behrisch, and E. Sackmann, Adhesive Switching of Membranes: Experiment and Theory, Phys. Rev. E 61, 4253 (2000).

[48] E. Sackmann and A. S. Smith, Physics of Cell Adhesion: Some Lessons from Cell-Mimetic Systems, Soft Matter 10, 1644 (2014).

[49] K. L. Wegener, A. W. Partridge, J. Han, A. R. Pickford, R. C. Liddington, M. H. Ginsberg, and I. D. Campbell,
Structural Basis of Integrin Activation by Talin, Cell 128, 171 (2007).

[50] S. Ghassemi, G. Meacci, S. Liu, A. A. Gondarenko, A. Mathur, P. Roca-Cusachs, M. P. Sheetz, and J. Hone, Cells Test Substrate Rigidity by Local Contractions on Submicrometer Pillars, Proc. Natl. Acad. Sci. U.S.A. 109, 5328 (2012).

[51] S. V. Plotnikov, A. M. Pasapera, B. Sabass, and C. M. Waterman, Force Fluctuations within Focal Adhesions Mediate ECM-Rigidity Sensing to Guide Directed Cell Migration, Cell 151, 1513 (2012).

[52] C. E. Chan and D. J. Odde, Traction Dynamics of Filopodia on Compliant Substrates, Science 322, 1687 (2008).

[53] R. McBeath, D. M. Pirone, C. M. Nelson, K. Bhadriraju, and C. S. Chen, Cell Shape, Cytoskeletal Tension, and RhoA Regulate Stem Cell Lineage Commitment, Dev. Cell 6, 483 (2004).

[54] M. A. Schwartz, Integrins and Extracellular Matrix in Mechanotransduction, Cold Spring Harbor Persp. Biol. 2, a005066 (2010).

[55] B. Trappmann et al., Extracellular-Matrix Tethering Regulates Stem-Cell Fate, Nat. Mater. 11, 642 (2012).

[56] J. H. Wen, L. G. Vincent, A. Fuhrmann, Y. S. Choi, K. C. Hribar, H. Taylor-Weiner, S. Chen, and A. J. Engler, Interplay of Matrix Stiffness and Protein Tethering in Stem Cell Differentiation, Nat. Mater. 13, 979 (2014).

[57] J. Du et al., Integrin Activation and Internalization on Soft ECM as a Mechanism of Induction of Stem Cell Differentiation by ECM Elasticity, Proc. Natl. Acad. Sci. U.S.A. 108, 9466 (2011).

[58] C. H. Yu, N. B. M. Rafiq, A. Krishnasamy, K. L. Hartman, G. E. Jones, A. D. Bershadsky, and M. P. Sheetz, IntegrinMatrix Clusters Form Podosome-like Adhesions in the Absence of Traction Forces, Cell biology international reports 5, 1456 (2013).

[59] A. Elosegui-Artola et al., Rigidity Sensing and Adaptation through Regulation of Integrin Types, Nat. Mater. 13, 631 (2014).

[60] C. H. Yu, J. B. K. Law, M. Suryana, H. Y. Low, and M. P. Sheetz, Early Integrin Binding to Arg-Gly-Asp Peptide Activates Actin Polymerization and Contractile Movement that Stimulates Outward Translocation, Proc. Natl. Acad. Sci. U.S.A. 108, 20585 (2011). 